Received 10 April 2015; accepted 31 May 2015. Date of publication 1 September 2015; date of current version 25 September 2015.

Digital Object Identifier 10.1109/JPETS.2015.2445296

\title{
Applications of Real-Time Simulation Technologies in Power and Energy Systems
}

\author{
IEEE PES Task Force ${ }^{1}$ on Real-Time Simulation of Power and Energy Systems \\ XAVIER GUILLAUD ${ }^{1}$ (Member, IEEE), M. OMAR FARUQUE² (Senior Member, IEEE), \\ ALEXANDRE TENINGE ${ }^{3}$, ALI HASAN HARIRI ${ }^{2}$ (Student Member, IEEE), \\ LUIGI VANFRETTII ${ }^{4}$ (Member, IEEE), MARIO PAOLONE ${ }^{5}$ (Senior Member, IEEE), \\ VENKATA DINAVAHI ${ }^{6}$ (Senior Member, IEEE), PINAKI MITRA ${ }^{7}$ (Member, IEEE), \\ GEORG LAUSS $^{8}$ (Member, IEEE), CHRISTIAN DUFOUR ${ }^{9}$ (Member, IEEE), \\ PAUL FORSYTH ${ }^{10}$ (Member, IEEE), \\ ANURAG K. SRIVASTAVA ${ }^{11}$ (Senior Member, IEEE), KAI STRUNZ ${ }^{12}$, \\ THOMAS STRASSER ${ }^{8}$ (Senior Member, IEEE), AND ALI DAVOUDI ${ }^{13}$ (Member, IEEE) \\ ${ }_{1}^{1}$ Power Electronic Laboratory, Department of Electrical Engineering, École Centrale de Lille, Villeneuve-d'Ascq 59651, France \\ ${ }^{2}$ Center for Advanced Power Systems, Department of Electrical and Computer Engineering, \\ Florida State University, Tallahassee, FL 32306 USA \\ ${ }^{3}$ Grenoble Electrical Engineering Laboratory, Grenoble 71348, France \\ ${ }^{4}$ KTH Royal Institute of Technology, Stockholm 114 28, Sweden \\ ${ }^{5}$ Swiss Federal Institute of Technology of Lausanne, Lausanne 1015, Switzerland \\ ${ }^{6}$ University of Alberta, Edmonton, AB T6G 2R3, Canada \\ ${ }^{7}$ Grid Systems Research and Development, ABB Corporate Research, Västerås 722 26, Sweden \\ ${ }^{8}$ Department of Electrical Energy Systems, AIT Austrian Institute of Technology, Vienna 1220, Austria \\ ${ }^{9}$ OPAL-RT Technologies Inc., Montréal, QC H3K 1G6, Canada \\ ${ }^{10}$ RTDS Technologies Inc., Winnipeg, MB R3T 2E1, Canada \\ ${ }^{11}$ Washington State University, Pullman, WA 99164 USA \\ ${ }^{12}$ Technische Universität Berlin, Elektrotechnik und Informatik, Berlin 10623, Germany \\ ${ }^{13}$ Department of Electrical Engineering, University of Texas at Arlington, Arlington, TX 76019 USA
}

CORRESPONDING AUTHOR: M. O. FARUQUE (faruque@ caps.fsu.edu)

\begin{abstract}
Real-time (RT) simulation is a highly reliable simulation method that is mostly based on electromagnetic transient simulation of complex systems comprising many domains. It is increasingly used in power and energy systems for both academic research and industrial applications. Due to the evolution of the computing power of RT simulators in recent years, new classes of applications and expanded fields of practice could now be addressed with RT simulation. This increase in computation power implies that models can be built more accurately and the whole simulation system gets closer to reality. This Task Force paper summarizes various applications of digital RT simulation technologies in the design, analysis, and testing of power and energy systems.
\end{abstract}

INDEX TERMS Applications, design, distribution networks, electric power circuits, hardware-in-theloop (HIL), modeling, rapid prototyping (RP), real-time (RT) simulation, testing, transmission networks.

\section{INTRODUCTION}

D IGITAL real-time (RT) simulators exploit advanced digital hardware and parallel computing methods to solve differential equations characterizing the system

\footnotetext{
${ }^{1}$ Task Force members: B. Asghari, J. Belanger, M. Bergeron, P. Beauchamp, E. Bernabeu, R. Brandl, M. Callavik, P. Crolla, A. Davoudi, V. Dinavahi, C. Dufour, R. Dugan, C. Edrington, Z. Fan, M. O. Faruque (Chair), S. Filizadeh, P. Forsyth, B. Francois, D. Geibel, X. Guillard, R. Iravani, V. Jalili, 'Panos Kotsampopoulos, M. Kuschke, J. Langston, C. Laros, G. Lauss, F. Lehfuss, J. Liu, W. Liu, T. Maguire, J. Mahseredjian, J. A. Martinez, M. Matar, P. Mclaren, A. Meherizi, Z. Miao, C. Muller, C. Nakazawa, J. L. Naredo, J. Peng, M. Saeedifard, K. Schoder, D. Sebastien, K. Sen, D. Shearer, E. Stein, M. Steurer, T. Strasser, K. Strunz, L. Vanfretti, A. Viehweider, X. Wang, and G. Zweigle were part of the Task Force on Real-Time Simulation Technologies.
}

behavior at a pace of real-world clock time. What differentiates digital RT simulations (DRTS) from non-RT or offline simulation platforms is the execution time of the simulation, i.e., the time it takes to solve the system equations and deliver back the output results. An RT simulator needs to solve the model equations for one time step within the same time in a real-world clock [1].

The RT simulation of electrical networks is not a new idea. A long time ago, it was the only means of simulation (known as analog RT simulator or transient network analyzer) with actual devices but in reduced size (i.e., lines were simulated with several set of pi-section devices composed of two capacitors and one inductance or with a set of operational amplifiers). Then, the hybrid simulators 
were developed where the most difficult devices, such as synchronous machines with their control, were implemented with digital simulators connected to the analog part with the amplifiers. With the evolution of the computer, the fully digital simulators started by the end of the 1990s [2].

Formerly, applications of RT simulations were mainly orientated toward transmission grid needs such as protection systems tests, thyristor-based high-voltage direct current (HVdc), or static VAR compensator (SVC) hardware control validation. Nowadays, RT simulation has also moved to distribution system applications due to the development of smart grid and microgrid studies. However, due to the short length of feeder lines and the presence of very high-frequency power electronic converters, RT simulation of a comparatively large modern distribution networks is much more challenging. The topology of some power electronic converters is becoming more and more complex. For example, modular multilevel converter (MMC)-based systems need to deal with enormous numbers of inputs/outputs. The hardware-in-theloop (HIL) simulation of such systems requires innovative approaches to deal with hundreds/thousands of gate pulses for the insulated-gate bipolar transistors or switches used in the converters. In addition, the HIL interface needs to have the required bandwidth and fidelity to generate the required voltage to apply to the devices under test or hardware under test (HUT).

This paper gives a summary of the applications of RT simulation in power and energy areas. It aims to gather most of the common applications that span from general to specific applications. These go all the way from power systems to power electronics to electric machines to other energy domains such as thermal, mechanical, fluid dynamics, and even water distribution systems. Moreover, in each of these fields, the applications vary between design, analysis, and testing. In this paper, beyond the electromagnetic transient (EMT) simulation, other types of RT simulation applications, such as phasor or hybrid simulation, are also covered. This paper attempts to provide a summary of RT simulation applications currently reported in the literature.

The high-level classification of applications of RT simulators is as follows; keep in mind that this is not a complete list but rather serves to offer a wide range of examples:

1) Functional applications:
a) Designing;
b) Rapid prototyping (RP);
c) Testing;
d) Teaching and training.

2) Field-specific applications:
a) Power systems;
b) Power electronics;
c) Control systems.

3) Simulation fidelity-based applications:
a) EMT simulation;
b) Phasor simulation;
c) Hybrid (phasor and EMT).

4) Multiphysics applications:

a) Thermoelectric;

b) Electromechanical;

c) Others.

Based on the setup of the simulation and its application, RT simulation can be classified into three main categories: 1) fully digital simulation; 2) controller HIL (CHIL) simulation; and 3) power HIL (PHIL) simulation. The characteristics of each type of RT simulation are described in the previous work of this Task Force [1].

\section{FUNCTIONAL APPLICATIONS \\ OF REAL-TIME SIMULATION}

This section highlights the functional applications of RT simulator in designing, prototype building, testing, teaching, and training.

\section{A. DESIGN AND MODELING}

The RT simulators can be used for developing models and designing a new concept/device for various applications. When built and simulated in RT, models will produce faster simulation results with higher accuracy compared with an offline simulation tool. In the designing stage, a fully DRTS mode is used. For industrial applications, the total time of product development is very important for competitiveness and reduction of the cost. Faster modeling and simulation can lead to a more efficient design process.

Fig. 1 shows the widely known $V$-curve with the application of DRTS (shown as RT) at various stages of the product development in the industry.

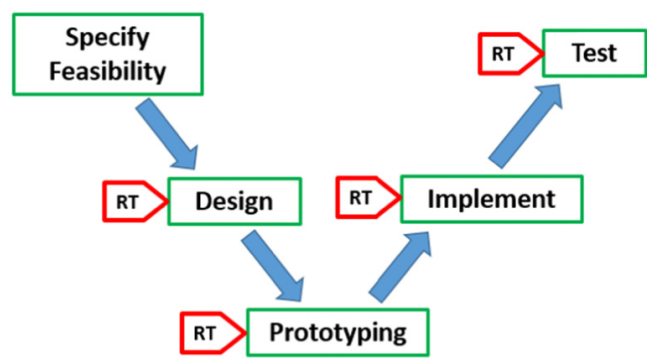

FIGURE 1. V-diagram illustrating the application of RT simulation at various stages of product development.

Among them, design is the first stage and there are many instances where DRTS is used for various design applications. In this paper, only a few are mentioned as example cases. The DRTS is used for modeling of an industrial 200-hp dc drive [3]; for developing new average models for two flexible-ac-transmission-system (FACTS) devices, SVC and static VAR generator [4]; for developing an accurate model of an inductive superconducting fault current limiter [5]; and for verification of a developed model of a submarine charging generator with a digital automatic voltage regulator (AVR) [6]. Many other examples can be found in the literature where the need to have accurate models 
in the design stage of different applications is of paramount importance to conduct more accurate and precise experiments at later stages. Some contemporary fields where DRTS is used for design purposes are photovoltaic generation system models [7], wind energy conversion systems [8], [9], and electric ship research [10].

\section{B. PROTOTYPING OR RAPID PROTOTYPING}

As seen in Fig. 1, DRTS is also used in the prototyping stage and its implementation. A prototype is an approximation of the real system that can be tested and modified as necessary. RP is becoming widely used in RT applications. Most commercial RT simulators use an automatic code generator that works as a transition between system modeling and system implementation [11]. This generated code facilitates the implementation of a rapid prototype which is then used in the integration and verification testing of the designed model [11].

Rapid control prototyping (RCP) is becoming more popular where the controller is built using the DRTS and is connected to a physical device. This technique offers more flexibility over fixing, debugging, tuning, and modifying the controller. The DRTS is being used for different prototyping and RP applications. For example, DRTS is used for RCP of a direct torque control of an induction motor-based on the HIL simulation [12]; prototyping a very high-speed closed-loop control of a virtual permanent magnet synchronous motor drive implemented on a field-programmable gate array [13]; prototyping and validation of a static synchronous compensation (STATCOM) by CHIL testing for a 10-MVA STATCOM for voltage regulation at the point of common coupling of a 50-MW wind farm [14]; and so on. In addition, many other applications in HIL experiments test prototypes of controllers and physical equipment before implementing and testing the commercial products.

\section{TESTING}

The most common application of DRTS is found to be testing. At the end of building the prototype or an actual full-scale device, it is time to test the device under RT conditions. Testing using DRTS (in HIL mode) provides added advantages over the laboratory testing in the sense that it allows to model the surrounding environments representing real physical field environments. Therefore, testing in RT is one of the major functional applications for RT simulators which provides close reproduction of how the equipment or product will behave in the real field. Many different examples are available in the literature to support the use of RT simulations for testing purposes. For instance, DRTS was used for testing:

1) certain relays for power system applications where a numeric distance relay with a new positive sequence directional element has been implemented and tested using HIL [15];

2) a controller for a long-stator linear synchronous motor using RT simulation platform [16];
3) a controller for multibus microgrid system [17];

4) a $500-\mathrm{kW}$ inverter in PHIL mode for volt/var control and other advanced functionality using a the model of field circuit data where it would be placed [18];

5) a megawatt level generator producing power at a frequency of $1.5 \mathrm{KHz}$ at $15000 \mathrm{r} / \mathrm{min}$ [19] in PHIL mode;

6) a 5-MW high-temperature superconducting rotating machine to assess the ac loss in the winding of the rotor circuit [20];

7) many other unique applications like HIL controller testing for spacecraft applications, in particular, testing of Odin, a three-axis stabilized small satellite for the Canadian Space Agency, altitude control system before it is implemented into the spacecraft [21].

Many of these testing applications may, otherwise, be impossible in a regular testing facility unless designed for that.

\section{TEACHING AND TRAINING}

Education is a major area that needs to be constantly updated in order to keep up with the industrial and technological advancements. To bridge the gap between education and industry, future engineers must be taught and trained using multidisciplinary approaches that cultivate creativity and simulate real-life conditions. Interactive simulations based on an RT simulator is becoming a valuable teaching tool that provides students with live feedback, allowing them to experience how systems react to applied changes [11].

The DRTS has many applications in the modern engineering education such as in: 1) electric machine drives; 2) mechatronics; and 3 ) electronic circuits and networks [22]. The DRTS is also applied in process dynamics and control education of junior chemical engineers and students [23]. In addition to classroom use, the DRTS is also used for engineers, operators, and technicians training, as shown in Fig. 2. This allows the user to become familiar with the controller and plant in the real system. DRTS is also very popular in pilot evaluation and training [24], as well as medical training that allows surgeons to test out multiple operations for a given problem [25], and so on.

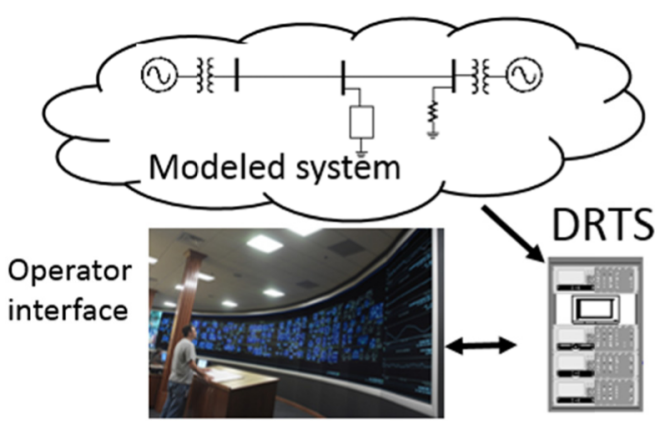

FIGURE 2. Operator and technician training concept. 


\section{TRANSMISSION SYSTEM APPLICATIONS}

DRTS of transmission systems started during the 1990s. The fields of interest for RT simulation applications in the transmission system are very wide. It could be used for many applications, such as improving the protection defense strategies with the aim to avoid voltage collapse or frequency instability, improving integration of power electronic devices to support voltage, and to test integration of large FACTS/HVdc transmission link. It is also used to assess the impact of large integration of renewable power generation in the grid [26].

The modeling of a transmission system is particularly suited for partitioning the network model into pieces for the purpose of allocation to multiple processors to reach parallel computation of nodal voltages. In this context, RT simulation takes advantage of the natural propagation delay of the traveling waves to split the network model at transmission lines where the propagation time exceeds the time step size of the simulation. This technique is used in all RT simulators and necessitates the modeling of transmission lines based on the distributed parameters. In most cases, the decoupling has to be done manually by the operator; however, there exists simulators with the capability of automatically decoupling the system at the most computationally appropriate node such as the one in [27].

\section{A. SUPERLARGE EMT REAL-TIME SIMULATION}

The RT simulation in EMT mode of superlarge transmission system including lines, transformers [with or without on-load tap changer (OLTC)], generators and their turbine (with governor and AVR), and loads, is a big challenge due to the high computational resources needed. To perform this kind of simulation, parallel processing is needed. One remarkable application is presented in [27] where a large part of the Hydro-Québec power system was modeled and simulated in RT. Major components of the model are as follows:

1) 643 three-phase buses;

2) 34 hydroelectric generators (turbine, AVR, and stabilizer);

3) one steam turbine generators;

4) 25 wind power plants (WPPs);

5) seven SVCs;

6) six synchronous condensers;

7) 167 three-phase lines;

8) 150 or more transformers including magnetic saturation.

Modeling of large WPPs is also presented with a decoupling technique for parallel processing (delay of transmission line not being always enough, this technique consists of moving and grouping line capacitor or transformer inductance in certain conditions). Then, an aggregation technique for large WPPs [28]-[30] is validated. This model could be used to study possible interactions between the series-compensated power system, real HVdc controls, and massive wind power generation. The RT simulation was realized using a 72-processor supercomputer with a 50- $\mu \mathrm{s}$ time step. This application shows the requirement of high computational resources. For economic constraints, computational resources could be limited which leads to limiting the size of the model. In this case, a part of the power system could be reduced with the aggregation of an area regrouping coherent generators [31] (area where generators have the tendency to swing together). Similar EMT-type large-scale RT simulations are carried out in different hardware simulator platforms where methods are developed to create large-scale models that can handle large numbers of breakers and phasor measurement units (PMUs) for testing of PMUs [32].

\section{B. PROTECTION, DEVICE INTEROPERABILITY, AND PMU}

The HIL feature of RT simulation provides a good arrangement to develop and to test new protection features and the device interoperability use in the protection chain. A setup was used to run RT tests on a suit of relays in a substation with a communication link to a remote control, as shown in Fig. 3, is presented in [33].

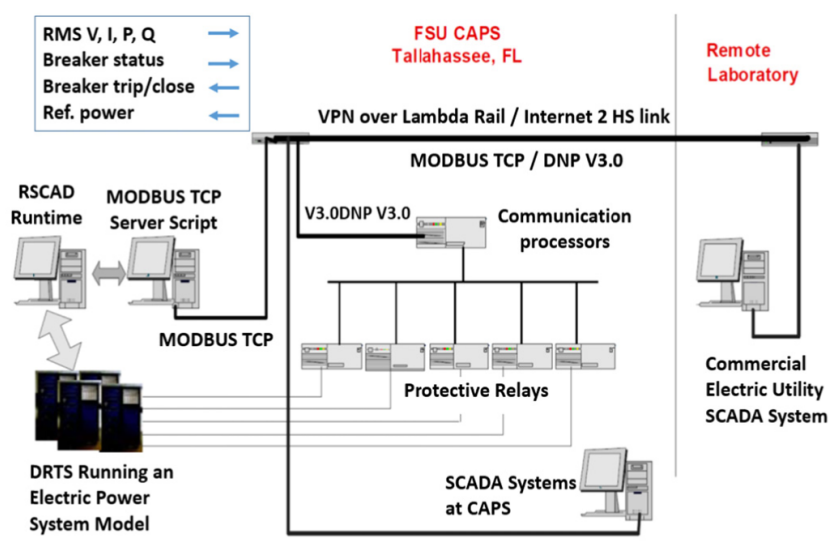

FIGURE 3. Diagram representation of a setup for performing tests on a suite of relays at the substation [33].

Similar work reported in [34] describes an RT environment to test the device protection and control schemes. It is focused on features and capabilities required for testing wide area protection and control based on synchrophasor data provided by PMUs with several communication standards and protocols (e.g., IEC 61850, C37.118, or DNP3). The development on an entirely software-based synchronized PMU for use within RT simulators to emulate a large number of real-life PMUs, is described in [35].

\section{HYBRID PHASOR/EMT APPLICATIONS}

Dynamic phasor simulation tools, also known as transient stability (TS) programs or TS analysis (TSA) programs, devoted to the studies of voltage control, voltage stability, or electromechanical TS do not need the same accuracy as for EMT studies. In those cases, phasor simulation deals with only fundamental frequency $(50 / 60 \mathrm{~Hz})$ and operates with a typical 8-10-ms time step. Solutions for RT simulation in phasor domain is not common in scientific research but was used for several years in dispatcher training simulator [36]. 
Recently, a solution has been developed in [37]. The use of both EMT and TS simulations has an interest in some cases [38]-[40], which are as follows.

1) The lack of computational resources for the simulation of a large-scale power system. In this case, only the part of the power system which is of great interest (as FACTS or HVdc) can be modeled in EMT, and the remainder in phasor domain.

2) The simulation of outages or blackouts where scale-bridging phenomena from electromechanical to EMTs is suitable for the combination of EMT and TS [41].

3) The need to use HIL with a system simulated in phasor mode [42].

However, the hybrid simulation is possible by accepting reduced accuracy for fast transient events that could occur in the EMT model. In the TS simulation, high-frequency components are ignored. Thus, there is a loss of information on electrical signals between the EMT area and the phasor area, which is a compromise that needs to be accepted. This is in addition to the fact that phasor model and EMT model operate in multirate, typically $10 \mathrm{~ms}$ and $50 \mu \mathrm{s}$, respectively. Multirate including delays between the models may produce large error in some cases of fast transients.

As presented in [39], [47], and [48], the accuracy of hybrid EMT-TS may look acceptable for an ac system; however, it is not always the case when ac/dc system is considered. These papers present a solution to improve the accuracy of the hybrid EMT-TS simulation with the ac/dc system. The solution is based on the integration of a frequency-dependent network equivalent (FDNE) at the interface between the EMT area and the phasor area. The role of the FDNE is to preserve the fast transient response of the power system by developing equivalents. The FDNE is obtained by fitting to the frequency response characteristic of the original network and it is modeled with a frequency-dependent admittance $[Y(f)]$ [39]. This permits to consider the high-frequency component, which is ignored in the TSA solution.

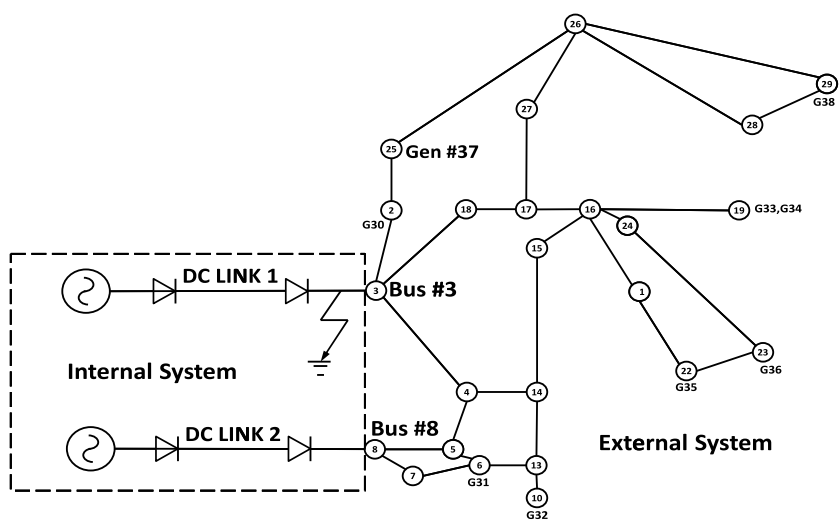

FIGURE 4. 39-bus New England ac system with two HVdc links [39].

In Fig. 4, a hybrid RT simulation of the New-England 39-bus ac system with two HVdc links is presented. The full detailed model is used for HVdc link, while the ac power system is modeled in phasor. Results present the comparison between EMT full model, EMT-TSA, and EMT-FDNE-TSA. The improvement in accuracy due to the FDNE application for hybrid simulation is clear in Fig. 5.

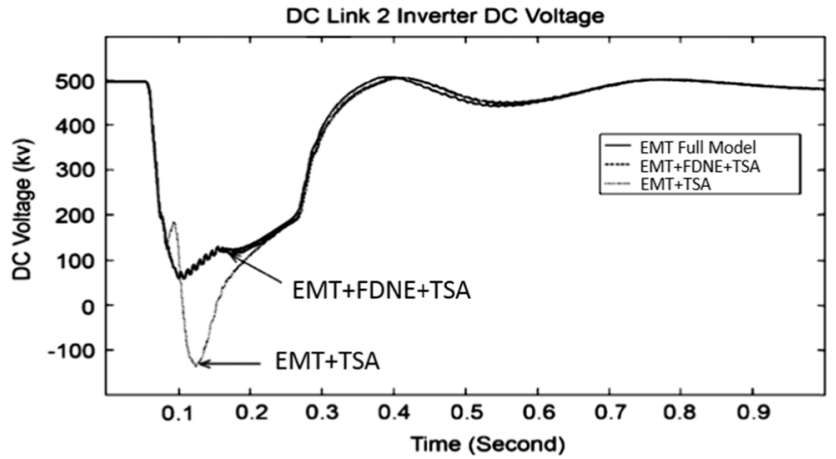

FIGURE 5. Comparison of the EMT-FDNE-TSA and EMT-TSA versus full real-time digital simulator (RTDS) simulation [39].

\section{HVdC TESTING}

In [49], an HVdc circuit composed of two back-to-back 12-pulse thyristor bridges was tested in RT simulation (Fig. 6). To test the proposed method using the DRTS environment, the two 12 pulse bridges were encapsulated in two user coded blocks. In this example, the two 12 pulse bridges are executed with a 10- $\mu$ s time step, while the rest of the model used 50- $\mu$ s time step. In [50], the same power electronic HVdc topology with switched filter bank is simulated with a $15-\mu$ s time step. Another alternative for fast simulation of $\mathrm{HVdc}$ is through the generation of exact Norton equivalents of the converters as developed in [51].

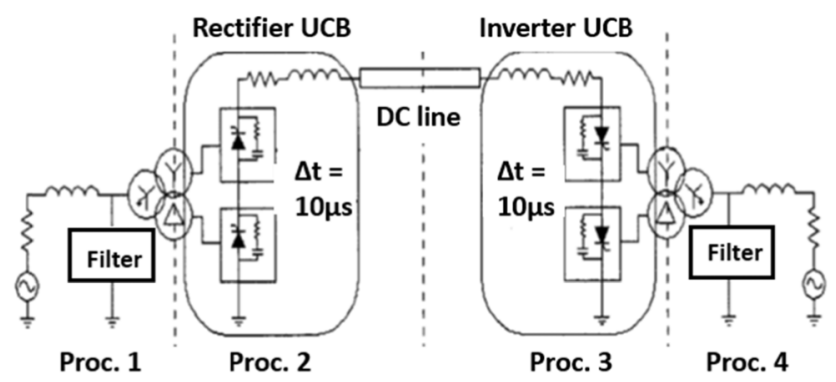

FIGURE 6. HVdc test circuit [49].

RT simulation was also used to evaluate the replacement of the Japanese Hokkaido-Honshu HVdc link control and protection system from an analog one to a digital one [52]. In this case, two manufacturers were in charge of one converter station; the RT simulation permits to check if each control system operates properly when they were combined together. The $\pm 250-\mathrm{kV}, 600-\mathrm{MW}$ HVdc link was implemented in the RT simulator, while the new real control, protection, and communication systems were tested using HIL setup.

In ABB's dc grid simulation center, HIL RT simulations are carried out for a three-terminal voltage source converter- 
based dc grid with symmetric monopole configuration (Fig. 7).

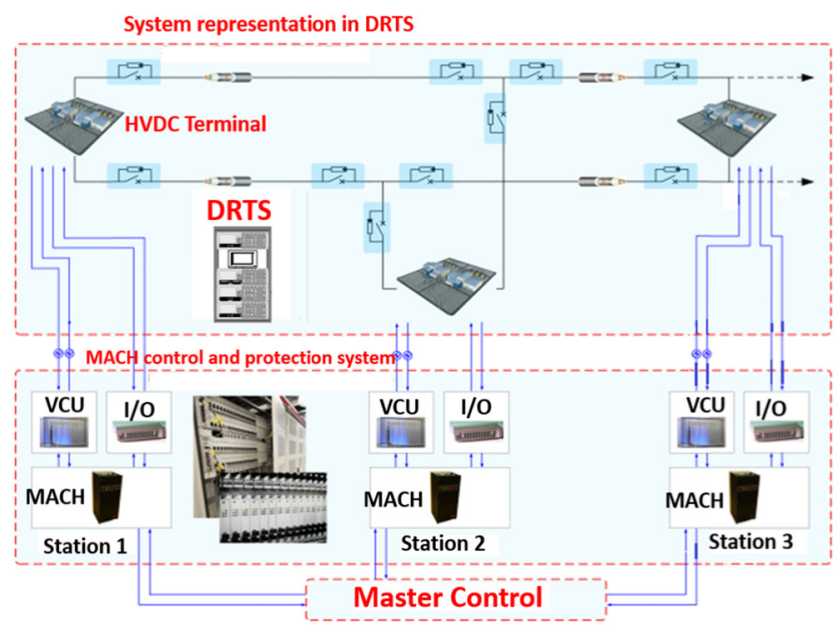

FIGURE 7. HIL simulation diagram for testing HVdc at ABB facility.

Here, the converters with cascaded two-level topology, the ac network equivalents, transformers, cables, and $\mathrm{dc}$ switchyard including dc breakers are all modeled inside an RT simulator [53]. However, for control and protection, ABB's MACH control and protection platform is used, so that the system behaves as close to reality as possible. Different measurement signals are sent out from DRTS to the MACH system through analog and digital I/O cards and at the same time the MACH system sends switching orders to the converter valves and breakers modeled inside the DRTS, being completely unaware of the fact that it is controlling a simulated system and not a real one. The HIL RT simulation naturally includes all communication delays which are important while performing fast control and protection actions. The dc grid simulation center is a powerful platform to demonstrate ABB's control and protection functions for $\mathrm{dc}$ grids including dc breakers and also to support research and development in that area.

\section{E. FACTS AND D-FACTS TESTING}

RT simulation is useful to improve the integration of power electronic devices in power systems. FACTS or D-FACTS offer flexible solutions to facilitate the electrical network exploitation. Without including details, we could mention the following RT applications:

1) D-STATCOM for voltage regulation [40], [54];

2) FACTS controller for damping interarea oscillation, with a thyristor-controlled series capacitor on a five area transmission power system [55];

3) use of an SVC for load compensation [56];

4) improving fault ride through of a WPP based on DFIG wind turbine using an STATCOM [57].

\section{F. MODULAR MULTILEVEL CONVERTER}

The MMC structures are composed from several hundreds to thousands of half-bridge or full-bridge converters. Such a large number of power switches and electrical nodes introduce important computational difficulties in EMT-type programs and thus in RT simulation. RT simulation of MMC systems is a major challenge due to the large numbers of submodules and I/O requirements for HIL testing. To solve this problem the MMC model needs to be simplified. Venjakob et al. [58] and Saad et al. [59] present different ways to match with the RT simulation of an MMC.

In [58], the equivalent MMC model assumes balanced submodules within each phase module, meaning that the algorithm will calculate only the average capacitor voltage. The state-space and nodal (SSN) method developed in [59] is another method used for the RT simulation of MMC. Fig. 8 shows the developed SSN-MMC model which uses the Norton equivalent model to represent each MMC arm. Another approach for simplified modeling of MMC using a surrogate network approach is discussed in [60]; it uses a modified topology to produce the same computational results but with less computational burden.

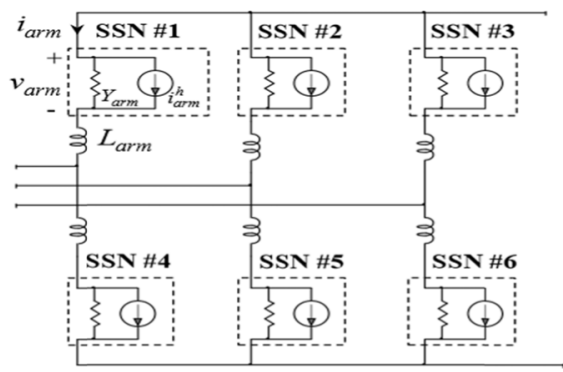

FIGURE 8. SSN-MMC model used in RT simulation [59].

\section{DISTRIBUTION SYSTEM/SMART \\ GRID APPLICATIONS}

The RT simulation of distribution networks is very challenging in the EMT mode. Distribution systems are composed of a small length line, thus propagation delays are pretty short (far less than the classical time step of $50 \mu \mathrm{s}$ generally used for EMT simulation) and the classical method based on this delay is not appropriated to solve the model on several computational units [61]. Nowadays, a multicore parallel solver based on an SSN solver is proposed in [62]. This solver allows the simulation of a model on parallel cores without adding any artificial delay. This solver offers a good solution even if there is currently no rule to design the model for its optimal use.

In most cases of distribution network studies, fast transient behaviors are not the concern, and thus phasor RT simulation may be appropriate to allow the simulation of large-scale distribution network. This type of simulation using RT has many applications for distribution systems.

\section{A. SMART GRID: VOLTAGE REGULATION WITH DISTRIBUTED GENERATION (DG) INTEGRATION (EMT SIMULATION)}

Development of an automatic distributed voltage control (ADVC) approach based on sensitivity theory was 
tested in [63]. This paper was done as part of the smart grids pilot project in collaboration with Enel Distribuzione S.p.A [the major Italian distribution system operator (DSO)]. The ADVC was tested on a 45-node MV distribution network using a DRTS. Another study which investigates a decentralized method for voltage control on a mediumscale distribution network with distributed generators (DGs) integration is presented in [61] and [64]. The present application used a multiagent system (MAS) to manage the reactive power of DGs [64]. The HIL RT experiment is shown in Fig. 9.

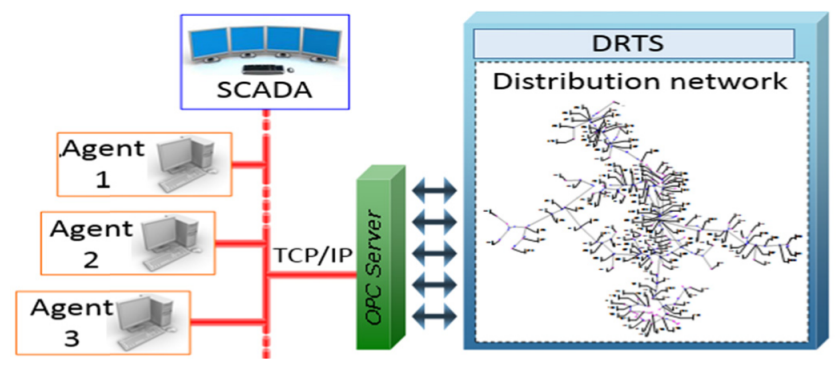

FIGURE 9. RT experiment using MAS and phasor RT simulation of a distribution network.

The distribution network has one OLTC transformer and five feeders for a total of 210 three-phase buses and 121 three-phase loads. The network was modeled in an EMT environment and is executed on six CPUs with a 50- $\mu$ s time step. A compensation method was developed to drastically reduce the error induced by the communication delay between CPUs. On the other hand, the MAS was developed in the Jade environment and the agents were executed on several computers. Data are exchanged between the agents and the RT simulator through an object linking and embedding (OLE) for process control (OPC) server and with the use of the transmission control protocol/Internet protocol (TCP/IP) communication protocol.

\section{B. SMART GRID: SUPERVISION STRATEGY FOR ELECTRIC VEHICLE CHARGING STATION (PHASOR SIMULATION)}

A recent application using an RT simulation in phasor type modeling is presented in [42]. This experimentation was developed to test a supervision strategy of a distribution network including DGs and a real electric vehicle (EV) charging station. The platform is also equipped with smart meters, communication systems, and a server. The whole system structure is shown in Fig. 10. Bouallaga et al. [42] investigate a supervision strategy which has the objectives to control EV load in order to limit the energy transmission costs of the DSO. The $15-\mathrm{kV}$ distribution network used to test this supervisor under real conditions is modeled in phasor domain. This system is composed of 447 three-phase nodes and 361 three-phase lines. It could be noted here that for PHIL the coupling between phasor simulation and real electrical devices imposes the need to create an EMT interface in the simulator.

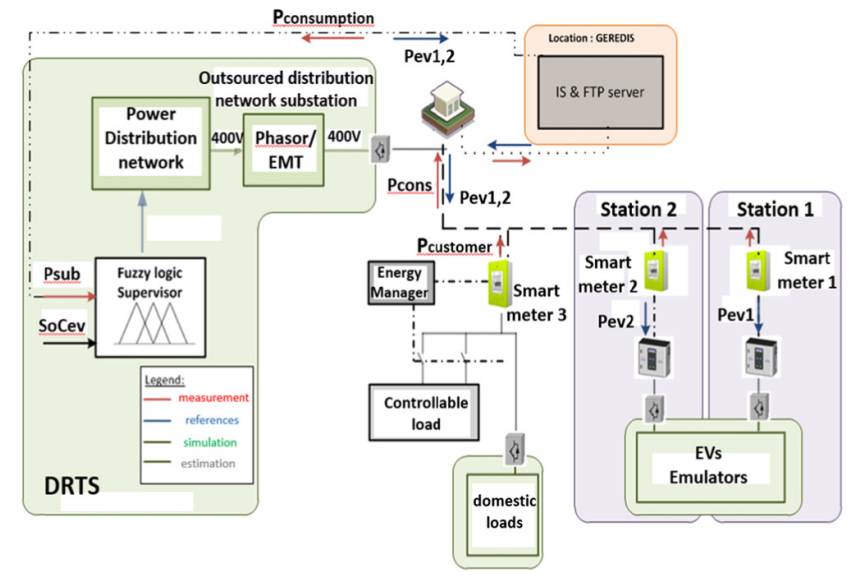

FIGURE 10. Distributed energy test platform overview for future distribution network research [42].

\section{PHIL SIMULATION OF ACTIVEIREACTIVE POWER CONTROL OF GRID-CONNECTED PHOTOVOLTAIC (PV) INVERTERS FOR AC VOLTAGE CONTROL CAPABILITIES}

One major issue of distribution systems is the active/reactive power control (PQ control) of the integrated generation units. It is currently part of many research projects and plays an important role in recent and upcoming grid connection standards [43]. The basic functions and parameter of a state-ofthe-art PQ control are well-known. They can be modeled in such a way that a PHIL simulation can simulate various grid configurations in RT (DRTS), while the grid-connected PV inverter (HUT) is interconnected. This simulation technique (PHIL) represents a massive simplification from hardware to software of both grid impedances and grid constellations resulting in flexibility and reduced costs.

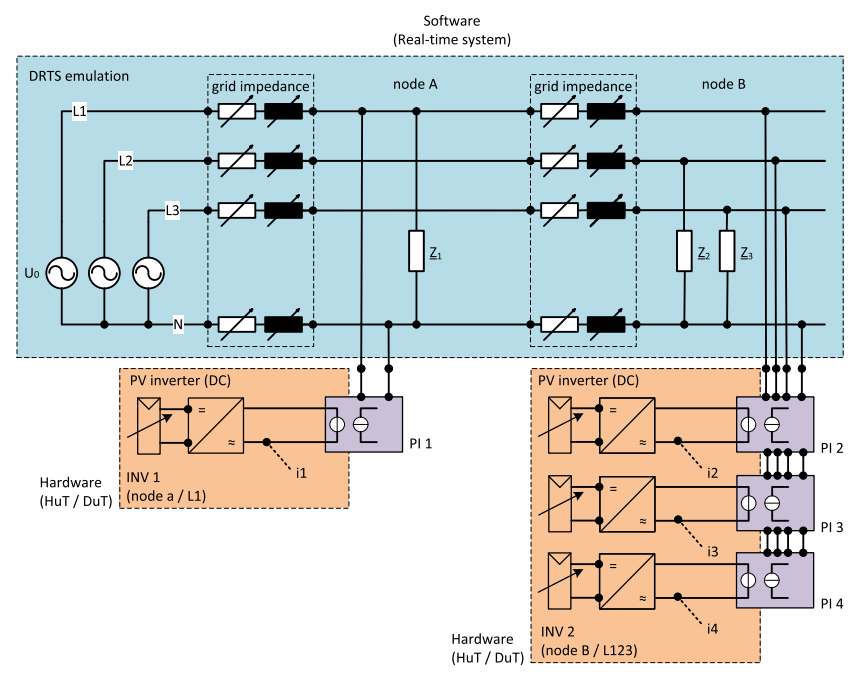

FIGURE 11. DRTS-based PHIL simulation test setup of grid-connected PV inverters to investigate active/ reactive power controls.

The RT modeling of the complex part of the distribution grid is indicated in the software (DRTS emulation) part in Fig. 11. The depicted test setup represents a fully 
adequate three-phase grid with two nodes, various (nonlinear) loads and two different connected PV inverters and four power interfaces (PI), respectively. In particular, the grid impedances vary heavily in value from one case to the other, thus a modification of their value is desired in software (DRTS) and not in hardware. The complexity of the emulated grid is only limited by the computation power of the DRTS and its minimum time step achievable (typically 50 or $10 \mu \mathrm{s}$ ). The sections labeled hardware in Fig. 11 represent the fixed installations of the PI as well as the PV inverters and its dc source.

Investigations on PQ controls [ $\cos \varphi(P), \cos \varphi(U), Q(U)$, $Q(P)$, static/dynamic, and free programmable controls] due to grid voltage or irradiance variations can be effectuated with sufficient high accuracy and simulation bandwidth $(>1 \mathrm{kHz})$. Observed field scenarios of oscillations or complete system instability can be reproduced via PHIL simulation and PQ control strategies, and solutions can be brought back to field applications. This would not be always possible with classical laboratory tests, simply because not every grid constellation in the field can be reproduced as easily as it can be done with the PHIL method. Further possible applications are located in the emulation of realistic grid fault scenarios and cosimulation approaches in smart grids.

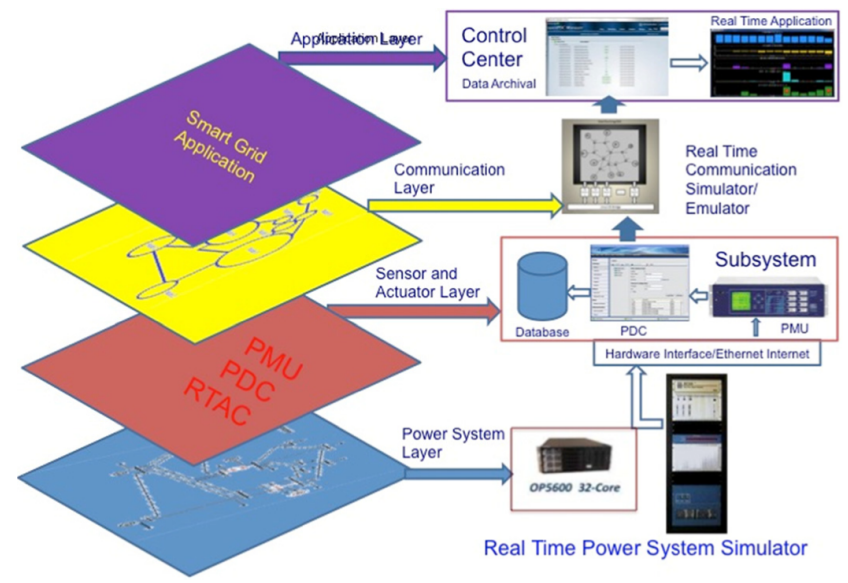

FIGURE 12. Example testbed for RT cyber physical analysis [44], [45].

\section{REAL-TIME CYBER PHYSICAL ANALYSIS FOR THE SMART GRID}

With the development of smart grid, new devices and applications need to be validated and analyzed for cyber-physical performance before the field installations. Cyber-physical simulation allows validation and analysis much closer to the real-life industry operating scenarios. Fig. 12 shows the testbed for the cyber physical analysis using RT modeling and simulation [44], [45]. This testbed has four layers to model the end-to-end system including RT power grid simulator, intelligent electronic devices (IEDs), data concentrators, communication emulators, and voltage stability applications. This testbed was used for the testing of wide area voltage stability applications and to analyze the impact of cyber events on the wide area voltage stability application.

\section{E. REAL-TIME OPERATION AND CONTROL OF MICROGRID}

Microgrids are one of the building blocks to realize the smart grid. A number of new algorithms for microgrid resiliency, reconfiguration, RT control, and operation are being developed which need to be validated and analyzed for RT implementation. Fig. 13 shows the testbed for RT modeling and simulation for testing the microgrid reconfiguration algorithm.

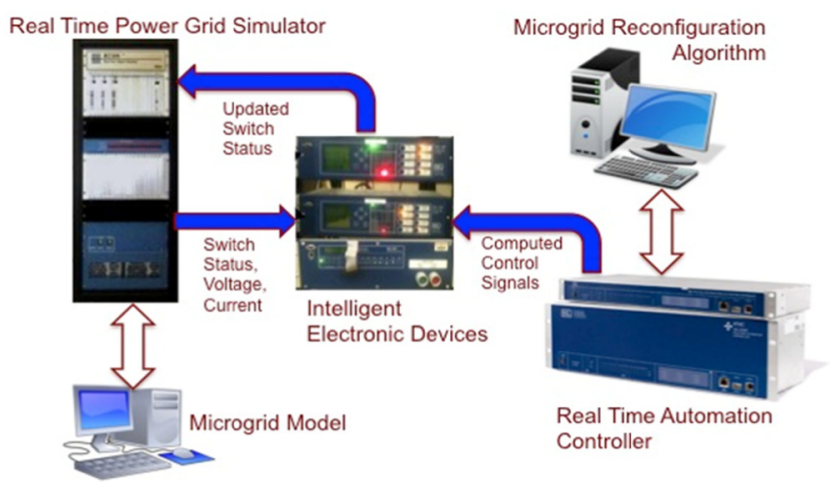

FIGURE 13. Example testbed for RT microgrid reconfiguration [46].

This testbed has a controller-in-the-loop running intelligent algorithm in the RT [46]. The microgrid was modeled using the RT power system simulator and IEDs were used to sense the input signals required for reconfiguration algorithm running in RT automation controller. The IEDs were also used to send the control signal back to power grid simulators. The particle swarm optimization-based reconfiguration algorithm was modified to run in RT and to analyze the performance of developed algorithm.

\section{MULTIPHYSICS APPLICATION}

\section{A. HOME ENERGY MANAGEMENT (EMT SIMULATION)}

As discussed in Section IV, distribution systems have undergone important changes. These changes lead to the development of smart grid system to keep the network operating safely while incorporating the advancement of technologies from other areas such as communication and information technology. Among solutions proposed in smart grid, one consists of the interest for DSOs in active demand side management involving the consumer. With this consideration, home is no longer a passive load, but rather an active controllable entity interacting with the grid. In parallel to that, the energy market will change and a dynamic pricing of energy will emerge with price and power subscription vary throughout the day. As a result, home energy management systems (HEMSs) will be developed. These systems should control a large panel of devices such as electrical loads and power production, e.g., PV, electrical storage system, and 
heater systems (electrical, domestic hot water, heat pump, or combined heat and power). The main goal of HEMS was to reduce the energy bill and to keep or to improve the human comfort [65], [66].

The RT simulation is a good solution to test the HEMS. In this context, the house has to be modeled, which implies a multiphysics modeling to consider the electrical and thermal behaviors or even hydraulic ones. In [65], a dwelling is modeled to test a building energy management system (BEMS) [67] in the RT domain. The thermal behavior, as the grid and some electrical devices, is modeled and executed on an RT simulator. On the other hand, the BEMS is implemented on an external computer and communicates with the RT simulator using TCP/IP protocol. Finally, a real heater was connected with a smart plug. The heater is electrically supplied by the modeled grid via a PHIL structure. The smart plug has the ability to provide the heat flow which is fed back to the simulation. It also has a switch and directly communicates with the BEMS using a wireless protocol. The test bench environment is shown in Fig. 14.

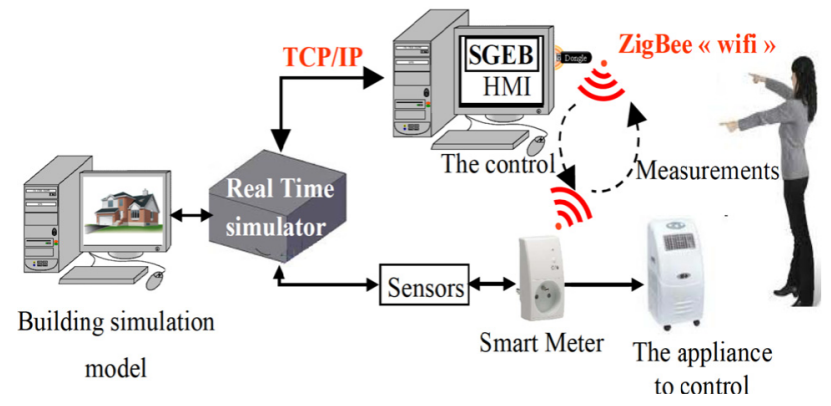

FIGURE 14. Test bench for BEMS in an RT domain [65].

A larger multiphysics testbed for renewable energy systems in smart homes is presented in [66]. The platform is, especially, designed for flexible multiphysics PHIL integrating three types of interface: 1) electrical; 2) thermal; and 3) hydraulic PI. The concept is shown in Fig. 15. It was realized to test home energy systems with buildings that include a heat pump, combined heat pump, water heat pump, electrical power generator, and/or battery storage system.

Here, the RT simulator is based on the personal computer (PC)-cluster (four nodes). One of the advantages of this PC-cluster is its ability to run different operating systems on each node. This is particularly important for the simulation of multiphysics systems which could ask different simulation tools.

The hydraulic interface could emulate the hydraulic part of combined heat pump, for instance. It is able to act as a heat source as well as a heat sink. The maximal heating/cooling load of this interface is $25 \mathrm{~kW}$.

The thermal interface is able to emulate the real temperature and humidity following accurately a given profile. It could be used to test air to water heat pump. This interface is realized by a climate chamber which could emulate realistic weather conditions. This interface can achieve a

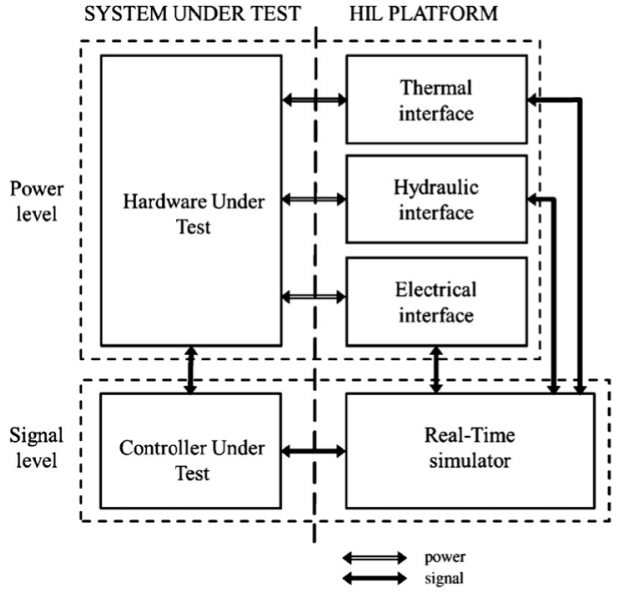

FIGURE 15. Concept of multiphysics PHIL platform [66].

flow rate of $2000 \mathrm{~L} / \mathrm{h}$ and the temperature range control is $10{ }^{\circ} \mathrm{C}-70^{\circ} \mathrm{C}$.

The electrical interface was developed to achieve great flexibility regarding the electrical connection of the HUT. The HUT may be a load or generator. This interface is based on a four-leg converter topology and allows testing on all kinds of unbalanced three-phase systems as well as two independent ac-single phase systems or two dc systems. This interface has a power rating of $25 \mathrm{~kW}$.

The example presented in [66] is a house of four rooms with a PV system and heated by an air to water heat pump. The building, the PV system, and the electrical loads, in addition to the heat pump, are simulated. In the scenario, the heat pump and the energy system interface are the HUT. The heating system of the investigated building has a nominal heating power of $9 \mathrm{~kW}$, a nominal electrical power rating of $2 \mathrm{~kW}$, and its flow rate is $1600 \mathrm{~L} / \mathrm{h}$.

\section{B. ELECTRIC SHIP: GEOGRAPHICALLY DISTRIBUTED THERMO-ELECTRIC COSIMULATION}

A case of thermoelectric cosimulation for electric ship was reported in [68] where a combined electrical and thermal simulation was carried out using two RT digital simulators

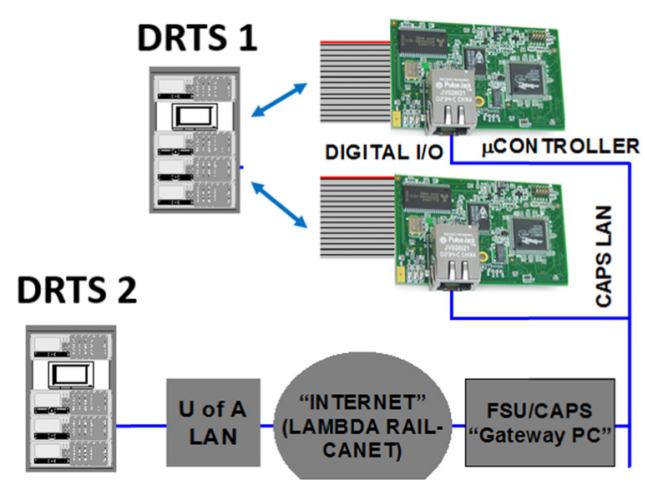

FIGURE 16. Thermoelectric cosimulation using two different DRTS [68]. 
located geographically apart from each other. The electrical model was developed on the first DRTS at the Center for Advanced Power Systems, Florida State University, Tallahassee, FL, USA, while the thermal model was developed on another DRTS of different type located at the RTX-Lab of the University of Alberta (U of A), Edmonton, $\mathrm{AB}$, Canada. The two simulators exchanged data in an asynchronous mode on the Internet utilizing the TCP/IP and user datagram protocol (UDP) protocols. Fig. 16 shows the setup of such cosimulation that may be feasible only when the two systems have time constants which are widely different.

\section{CONCLUSION}

This paper presented a thorough review of applications of RT simulation in different areas in power and energy systems. This paper categorizes the applications into four major classes such as functional applications, field-specific applications, simulation fidelity based applications, and multiphysical applications. It also differentiates between applications at the transmission system and distribution system, since each system has its own specifications and characteristics that require different types of solutions. The objective of this paper is to summarize its major applications so that readers can be familiar with the DRTS usage. The role of RT digital simulation in power systems is rapidly evolving and becoming more essential for industrial and academic research work. The DRTS will bring the testing and research environment closer to real life leading to more accurate and meaningful studies. Future possible application could dive deeper into power electronics modeling as this area is so far the most challenging due to the large number of computations needed in a very short period of time. Thus, more algorithms and faster hardware are yet to be developed to accommodate the challenges of smaller time steps and larger systems. The DRTS applications would soon extend beyond power and energy systems to fields such as aviation and medical surgery and many others.

\section{ACKNOWLEDGMENT}

Task Force on Real-Time Simulation Technologies for Power Systems Analysis is with the Working Group on Modeling and Analysis of System Transients Using Digital Programs, General Systems Sub-Committee, T\&D Committee, IEEE Power and Energy Society: B. Asghari (e-mail: b.asghari@gmail.com); J. Belanger (e-mail: jean.belanger@ opal-rt.com); M. Bergeron (e-mail: mathieu.bergeron@ power. alstom.com); P. Beauchamp (e-mail: Philippe.Beauchamp@ opal-rt.com); E. Bernabeu (e-mail: emanuel.e.bernabeu@dom .com); R. Brandl (e-mail: ron.brandl@iwes.fraunhofer.de); M. Callavik (e-mail: magnus.callavik@se.abb.com); P. Crolla (e-mail: paul.crolla@eee.strath.ac.uk); A. Davoudi (e-mail: davoudi@uta.edu); V. Dinavahi (e-mail: dinavahi@ ualberta.ca); C. Dufour (e-mail: christian.dufour@opalrt.com); R. Dugan (e-mail: r.dugan@ieee.org); C. Edrington (e-mail: edrington@caps.fsu.edu); Z. Fan (e-mail: ); M. O. Faruque (Chair) (e-mail: faruque@ caps.fsu.edu);
S. Filizadeh (e-mail: sfilizad@ece.umanitoba.ca); P. Forsyth (e-mail: paf@rtds.com); B. Francois (e-mail: francois. berthelot@opal-rt.com); D. Geibel (e-mail: dominik.geibel@ iwes.fraunhofer.de); X. Guillard (e-mail: xavier.guillaud@eclille.fr); R. Iravani (e-mail: iravani@ecf.utoronto.ca); V. Jalili (e-mail: vahidj@opal-rt.com); Panos Kotsampopoulos (e-mail: ); M. Kuschke (e-mail: maren.kuschke@ieee.org); J. Langston (e-mail: langston@caps.fsu.edu); C. Laros (e-mail: larose.christian@ireq.ca); G. Lauss (e-mail: georg. lauss@ait.ac.at); F. Lehfuss (e-mail: felix.lehfuss@ait.ac.at); J. Liu (e-mail: ljw@ieee.org); W. Liu (e-mail: wliu@nmsu. edu); T. Maguire (e-mail: tlm@rtds.com); J. Mahseredjian (e-mail: jean.mahseredjian@polymtl.ca); J. A. Martinez (e-mail: martinez@ee.upc.edu); M. Matar (e-mail: mah.matar @utoronto.ca); P. Mclaren (e-mail: mclaren@caps.fsu.edu); A. Meherizi (e-mail: mehrizi@eecs.wsu.edu); Z. Miao (e-mail: zmiao@usf.edu); C. Muller (e-mail: cmuller@ hvdc.ca); C. Nakazawa (e-mail: nakazawa-chikashi@ fujielectric.co.jp); M. Saeedifard (e-mail: maryam@ece. gatech.edu); K. Schoder (e-mail: schoder@caps.fsu.edu); D. Sebastien (e-mail: sebastien.dennetiere@ rte-france.com); K. Sen (e-mail: senkk@sentransformer.com); D. Shearer (e-mail: david.shearer@se.abb.com); E. Stein (e-mail: ); M. Steurer (e-mail: ); T. Strasser (e-mail: Thomas.Strasser@ ait.ac.at); K. Strunz (e-mail: kai.strunz@tu-berlin.de); L. Vanfretti (e-mail: luigiv@kth.se); A. Viehweider (e-mail: viehweider@ieee.org); and X. Wang (e-mail: Xiaoyu.Wang3 @ carleton.ca).

\section{REFERENCES}

[1] M. O. Faruque et al., "Real-time simulation technologies for power systems analysis, design and testing," IEEE Power Energy Technol. Syst. J., to be published.

[2] R. P. Wierckx, "Fully digital real-time electromagnetic transients simulator," in Proc. IERE Int. Electr. Res. Exchange, Workshop New Issues Power Syst. Simul., Caen, France, Mar. 1992, pp. 218-228.

[3] P. Terwiesch, T. Keller, and E. Scheiben, "Rail vehicle control system integration testing using digital hardware-in-the-loop simulation," IEEE Trans. Control Syst. Technol., vol. 7, no. 3, pp. 352-362, May 1999.

[4] I. Etxeberria-Otadui, V. Manzo, S. Bacha, and F. Baltes, "Generalized average modelling of FACTS for real time simulation in ARENE," in Proc. IEEE Annu. Conf. Ind. Electron. Soc. IECON, vol. 2. Nov. 2002, pp. 864-869.

[5] L. Graber, T. Chiocchio, J. Kvitkovic, S. Pamidi, M. Steurer, and A. Usoskin, "Finite element model of a superconducting fault current limiter calibrated by hardware-in-the-loop measurements," IEEE Trans. Appl. Supercond., vol. 24, no. 3, Jun. 2014, Art. ID 5601105.

[6] J.-H. Yoon, S.-H. Lee, J.-G. Bin, Y.-K. Kong, and S.-S. Lee, "The modeling and simulation for the design verification of submarine charging generator by using real-time simulator," in Proc. IEEE 8th Int. Conf. Power Electron. ECCE Asia, Jeju, Korea, May/Jun. 2011, pp. 1406-1410.

[7] M. Park and I.-K. Yu, "A novel real-time simulation technique of photovoltaic generation systems using RTDS," IEEE Trans. Energy Convers., vol. 19, no. 1, pp. 164-169, Mar. 2004.

[8] G. Wang and W. Gao, "Real time simulation for wind power generation system using RTDS," in Proc. 40th North Amer. Power Symp. (NAPS), Calgary, AB, Canada, Sep. 2008, pp. 1-6.

[9] L.-F. Pak and V. Dinavahi, "Real-time simulation of a wind energy system based on the doubly-fed induction generator," IEEE Trans. Power Syst., vol. 24, no. 3, pp. 1301-1309, Aug. 2009.

[10] W. Ren, M. Steurer, S. Woodruff, and P. F. Ribeiro, "Augmenting E-ship power system evaluation and converter controller design by means of realtime hardware-in-loop simulation," in Proc. IEEE Electr. Ship Technol. Symp., Jul. 2005, pp. 171-175. 
[11] J. Bélanger, P. Venne, and J.-N. Paquin, "The what, where and why of real-time simulation," in Proc. PES General Meeting, Oct. 2010, pp. $37-49$.

[12] S. Vamsidhar and B. G. Fernandes, "Hardware-in-the-loop simulation based design and experimental evaluation of DTC strategies," in Proc. IEEE Power Electron. Specialists Conf., Aachen, Germany, Jun. 2004, pp. 3615-3621.

[13] C. Dufour, H. Blanchette, and J. Belanger, "Very-high speed control of an FPGA-based finite-element-analysis permanent magnet synchronous virtual motor drive system," in Proc. IEEE 34th Annu. Conf. Ind. Electron. (IECON), Orlando, FL, USA, Nov. 2008, pp. 2411-2416.

[14] Y. Liu et al., "Controller hardware-in-the-loop validation for a $10 \mathrm{MVA}$ ETO-based STATCOM for wind farm application," in Proc. IEEE Energy Convers. Congr. Expo., 2009, pp. 1398-1403.

[15] P. G. McLaren, G. W. Swift, Z. Zhang, E. Dirks, R. P. Jayasinghe, and I. Fernando, "A new directional element for numerical distance relays," IEEE Trans. Power Del., vol. 10, no. 2, pp. 666-675, Apr. 1995.

[16] Y. Li, L. M. Shi, H. Zhang, and Y. Du, "Real-time simulation of linear synchronous motor in hardware-in-loop test system," in Proc. Int. Conf. Electr. Mach. Syst., Incheon, Korea, Oct. 2010, pp. 1520-1523.

[17] Y. Li, D. M. Vilathgamuwa, and P. C. Loh, "Design, analysis, and real-time testing of a controller for multibus microgrid system," IEEE Trans. Power Electron., vol. 19, no. 5, pp. 1195-1204, Sep. 2004

[18] J. Langston et al., "Power hardware-in-the-loop testing of a $500 \mathrm{~kW}$ photovoltaic array inverter," in Proc. IEEE Conf. Ind. Electron. (IECON), Montreal, QC, Canada, Oct. 2012, pp. 4797-4802.

[19] J. Langston et al., "Megawatt scale hardware-in-the-loop testing of a high speed generator," in Proc. Amer. Soc. Naval Eng. (ASNE), Feb. 2012, pp. $1-12$

[20] M. Steurer, S. Woodruff, H. Boenig, F. Bogdan, and M. Sloderbeck, "Hardware-in-the-loop experiments with a 5 MW HTS propulsion motor at Florida State University's power test facility," in Proc. IEEE PES Gen. Meeting, Jun. 2007, pp. 1-4.

[21] A. Ptak and K. Foundy, "Real-time spacecraft simulation and hardwarein-the-loop testing," in Proc. 4th IEEE Real-Time Technol. Appl. Symp., Denver, CO, USA, Jun. 1998, pp. 230-236.

[22] P. M. Menghal and A. J. Laxmi, "Real time simulation: A nove approach in engineering education," in Proc. Int. Conf. Electron. Comput. Technol. (ICECT), Apr. 2011, pp. 215-219.

[23] Y. Zhang, M. T. Munir, W. Yu, and B. R. Young, "A real time approach to process control education with an open-ended simulation tool," Edu. Chem. Eng., vol. 8, no. 3, pp. e105-e112, Aug. 2013.

[24] C. S. Tzafestas, K. Birbas, Y. Koumpouros, and D. Christopoulos, "Pilot evaluation study of a virtual paracentesis simulator for skill training and assessment: The beneficial effect of haptic display," Presence Teleoperators AMP Virtual Environ., vol. 17, no. 2, pp. 212-229, Apr. 2008.

[25] M. Akay and A. Marsh, Information Technologies in Medicine: Medical Simulation and Education, vol. 1. Hoboken, NJ, USA: Wiley, 2001.

[26] Q. Dacai, "Defence schema against large disturbances in China southern power grid," Electra, no. 257, pp. 4-16, Aug. 2011.

[27] R. Gagnon, G. Turmel, C. Larose, J. Brochu, G. Sybille, and M. Fecteau, "Large-scale real-time simulation of wind power plants into Hydro-Québec power system," in Proc. 9th Int. Workshop Large-Scale Integr. Wind Power Power Syst. Well Transmiss. Netw. Offshore Wind Plants, Quebec City, QC, Canada, Oct. 2010, pp. 1-8.

[28] E. Muljadi et al., "Equivalencing the collector system of a large wind power plant," in Proc. IEEE Power Eng. Soc. Gen. Meeting, Montreal, QC, Canada, Jun. 2006, pp. 1-8.

[29] E. Muljadi, S. Pasupulati, A. Ellis, and D. Kosterov, "Method of equivalencing for a large wind power plant with multiple turbine representation," in Proc. IEEE Power Eng. Soc. Gen. Meeting, Pittsburgh, PA, USA, Jul. 2008, pp. 1-9.

[30] V. Jalili-Marandi, L.-F. Pak, and V. Dinavahi, "Real-time simulation of grid-connected wind farms using physical aggregation," IEEE Trans. Ind. Electron., vol. 57, no. 9, pp. 3010-3021, Sep. 2010.

[31] M. L. Ourari, L.-A. Dessaint, and V. Q. Do, "Integration of dynamic equivalents in Hypersim power system simulator," in Proc. IEEE PES Gen. Meeting, Tampa, FL, USA, Jun. 2007, pp. 1-6.

[32] T. Bi, Y. Zhang, X. Xiao, P. Forsyth, and R. Wierckx, "Large scale power system simulation and PMU testing using a real time digital simulator," in Proc. Int. Power Eng. Conf., Dec. 2007, pp. 383-388.
[33] P. G. McLaren, J. Tang, D. Ouellette, M. Steurer, and R. Kuffel, "Using a real time simulator to design, prove and test power system protection systems," in Proc. Conf. Power Syst. (CIGRE), Halifax, NS, Canada, Sep. 2011.

[34] P. Forsyth, D. S. Ouellette, C. Peters, and M. D. Desjardine, "A real-time environment for closed-loop testing of PMU-based WACS schemes," in Proc. CIGRE, Calgary, AB, Canada, Sep. 2013.

[35] A. T. Al-Hammouri, L. Nordström, M. Chenine, L. Vanfretti, N. Honeth, and R. Leelaruji, "Virtualization of synchronized phasor measurement units within real-time simulators for smart grid applications," in Proc. PES Gen. Meeting, San Diego, CA, USA, Jul. 2012, pp. 1-7.

[36] S. Gissinger, P. Chaumes, J.-P. Antoine, A. Bihain, and M. Stubbe, "Advanced dispatcher training simulator," IEEE Comput. Appl. Power, vol. 13, no. 2, pp. 25-30, Apr. 2000.

[37] V. Jalili-Marandi, F. J. Ayres, E. Ghahremani, J. Belanger, and V. Lapointe, "A real-time dynamic simulation tool for transmission and distribution power systems," in Proc. IEEE PES Gen. Meeting, Vancouver, BC, Canada, Jul. 2013, pp. 1-5.

[38] V. Jalili-Marandi, F. J. Ayres, C. Dufour, and J. Bélanger, "Real-time electromagnetic and transient stability simulations for active distribution networks," in Proc. Int. Conf. Power Syst. Transients (IPTS), Vancouver, BC, Canada, Jul. 2013, pp. 1-7.

[39] X. Lin, A. M. Gole, and M. Yu, "A wide-band multi-port system equivalent for real-time digital power system simulators," IEEE Trans. Power Syst., vol. 24, no. 1, pp. 237-249, Feb. 2009.

[40] V. Jalili-Marandi, V. Dinavahi, K. Strunz, J. A. Martinez, and A. Ramirez, "Interfacing techniques for transient stability and electromagnetic transient programs IEEE task force on interfacing techniques for simulation tools," IEEE Trans. Power Del., vol. 24, no. 4, pp. 2385-2395, Oct. 2009.

[41] F. Gao and K. Strunz, "Frequency-adaptive power system modeling for multiscale simulation of transients," IEEE Trans. Power Syst., vol. 24, no. 2, pp. 561-571, May 2009.

[42] A. Bouallaga et al., "Advanced metering infrastructure for real-time coordination of renewable energy and electric vehicles charging in distribution grid," in Proc. CIRED Workshop, Rome, Italy, Jun. 2014, pp. 1-7.

[43] Erzeugungsanlagen am Niederspannungsnetz-Technische Mindestanforderungen für Anschluss und Parallelbetrieb von Erzeugungsanlagen am Niederspannungsnetz, Standard VDE-AR-N4105:2011-08.

[44] C. B. Vellaithurai, S. S. Biswas, R. Liu, and A. Srivastava, "Real time modeling and simulation of cyber-power system," in Cyber Physical Systems Approach to Smart Electric Power Grid. Berlin, Germany: Springer-Verlag, 2015, pp. 43-74.

[45] R. Liu, C. Vellaithurai, S. S. Biswas, T. T. Gamage, and A. K. Srivastava, "Analyzing the cyber-physical impact of cyber events on the power grid," IEEE Trans. Smart Grid, to be published.

[46] F. Shariatzadeh, C. B. Vellaithurai, S. S. Biswas, R. Zamora, and A. K. Srivastava, "Real-time implementation of intelligent reconfiguration algorithm for microgrid," IEEE Trans. Sustainable Energy, vol. 5, no. 2, pp. 598-607, Apr. 2014

[47] Y. Liang, X. Lin, A. M. Gole, M. Yu, Y. Zhang, and B. Zhang, "Comparisons of impact on the modeling detail on real time simulation of large power systems with HVDC," in Proc. Int. Conf. Power Syst. Transients (IPTS), Delft, The Netherlands, Jun. 2011, pp. 1-6.

[48] Y. Hu, W. Wu, B. Zhang, and Q. Guo, "Development of an RTDS-TSA hybrid transient simulation platform with frequency dependent network equivalents," in Proc. 4th IEEE/PES Innovative Smart Grid Technol. Eur. (ISGT EUROPE), Kongens Lyngby, Denmark, Oct. 2013, pp. 1-5.

[49] B. de Kelper, L. A. Dessaint, K. Al-Haddad, and H. Nakra, "A comprehensive approach to fixed-step simulation of switched circuits," IEEE Trans. Power Electron., vol. 17, no. 2, pp. 216-224, Mar. 2002

[50] C. Dufour, S. Abourida, J. Belanger, and V. Lapointe, "InfiniBandbased real-time simulation of HVDC, STATCOM, and SVC devices with commercial-off-the-shelf PCs and FPGAs," in Proc. IEEE 32nd Annu. Conf. Ind. Electron. (IECON), Paris, France, Nov. 2006, pp. 5325-5331.

[51] K. Strunz and E. Carlson, "Nested fast and simultaneous solution for timedomain simulation of integrative power-electric and electronic systems," IEEE Trans. Power Del., vol. 22, no. 1, pp. 277-287, Jan. 2007.

[52] H. Horii, Y. Kida, T. Ooishi, G. Managaki, F. Saito, and K. Takahashi, "Closed-loop test of HVDC control system using real-time digital simulator (RTDS)," in Proc. Int. Conf. Elect. Eng. (ICEE), Okinawa, Japan, Jul. 2008 
[53] P. Mitra and T. Larsson, "ABB's HVDC grid simulation center-A powerful tool for real-time research of DC grids," in Proc. EWEA Annu. Event, Barcelona, Spain, Mar. 2014, pp. 1-10.

[54] G. Sybille and P. Giroux, "Simulation of FACTS controllers using the MATLAB power system blockset and Hypersim real-time simulator," in Proc. IEEE PES Winter Meeting, New York, NY, USA, Jan. 2002, pp. $488-491$

[55] R. Majumder, B. C. Pal, C. Dufour, and P. Korba, "Design and real-time implementation of robust FACTS controller for damping inter-area oscillation," IEEE Trans. Power Syst., vol. 21, no. 2, pp. 809-816, May 2006.

[56] J. Berge and R. K. Varma, "Design and development of a static VAR compensator for load compensation using real-time digital simulator and hardware simulation," in Proc. Large Eng. Syst. Conf. Power Eng., Montreal, QC, Canada, Oct. 2007, pp. 6-12.

[57] W. Qiao, G. K. Venayagamoorthy, and R. G. Harley, "Real-time implementation of a STATCOM on a wind farm equipped with doubly fed induction generators," IEEE Trans. Ind. Appl., vol. 45, no. 1, pp. 98-107, Jan./Feb. 2009

[58] O. Venjakob, S. Kubera, R. Hibberts-Caswell, P. A. Forsyth, and T. L. Maguire, "Setup and performance of the real-time simulator used for hardware-in-loop-tests of a VSC based HVDC scheme for offshore applications," in Proc. Int. Conf. Power Syst. Transients (IPST), Vancouver, BC, Canada, Jul. 2013, pp. 18-20.

[59] H. Saad, C. Dufour, J. Mahseredjian, S. Dennetière, and S. Nguefeu, "Real time simulation of MMCs using the state-space nodal approach," in Proc. Int. Conf. Power Syst. Transients (IPST), Vancouver, BC, Canada, Jul. 2013, pp. 1-7.

[60] K. Ou et al., "MMC-HVDC simulation and testing based on real-time digital simulator and physical control system," IEEE J. Emerging Sel. Topics Power Electron., vol. 2, no. 4, pp. 1109-1116, Dec. 2014.

[61] A. Teninge, Y. Besanger, F. Colas, H. Fakham, and X. Guillaud, "Real-time simulation of a medium scale distribution network: Decoupling method for multi-CPU computation," in Proc. Complex. Eng. (COMPENG), Aachen, Germany, Jun. 2012, pp. 1-6.

[62] C. Dufour, V. Jalili-Marandi, J. Belanger, and L. Snider, "Power system simulation algorithms for parallel computer architectures," in Proc. IEEE PES Gen. Meeting, San Diego, CA, USA, Jul. 2012, pp. 1-6.

[63] M. Brenna et al., "Automatic distributed voltage control algorithm in smart grids applications," IEEE Trans. Smart Grid, vol. 4, no. 2, pp. 877-885, Jun. 2013.

[64] H. Fakham, A. Ahmidi, F. Colas, and X. Guillaud, "Multi-agent system for distributed voltage regulation of wind generators connected to distribution network," in Proc. IEEE PES Innovative Smart Grid Technol. Conf. Eur. (ISGT Europe), Gothenburg, Sweden, Oct. 2010, pp. 1-6.

[65] R. Missaoui, G. Warkozek, S. Bacha, and S. Ploix, "Real time validation of an optimization building energy management strategy based on power-hardware-in-the-loop tool," in Proc. IEEE PES Innovative Smart Grid Technol. Eur. (ISGT Europe), Berlin, Germany, Oct. 2012, pp. 1-7.

[66] C. Molitor et al., "Multiphysics test bed for renewable energy systems in smart homes," IEEE Trans. Ind. Electron., vol. 60, no. 3, pp. 1235-1248, Mar. 2013.

[67] D. L. Ha, H. Joumaa, S. Ploix, and M. Jacomino, "An optimal approach for electrical management problem in dwellings," Energy Buildings, vol. 45, pp. 1-14, Feb. 2012.

[68] M. O. O. Faruque, M. Sloderbeck, M. Steurer, and V. Dinavahi, "Thermoelectric co-simulation on geographically distributed real-time simulators," in Proc. IEEE PES Gen. Meeting, Calgary, AB, Canada, Jul. 2009, pp. 1-7.

XAVIER GUILLAUD (M'03) received the Ph.D. degree from the University of Lille, Villeneuve-d'Ascq, France, in 1992.

He joined the Laboratory of Electrical Engineering and Power Electronic, Lille, France, in 1993. He has been a Professor with the École Centrale de Lille, Villeneuve-d'Ascq, France, since 2002. His current research interests include the integration of high voltage power electronic converters in transmission system.
M. OMAR FARUQUE (S'03-M'08-SM'14) received the Ph.D. degree from the University of Alberta, Edmonton, AB, Canada, in 2008.

$\mathrm{He}$ is with the Center for Advanced Power Systems and the Department of Electrical and Computer Engineering, Florida State University, Tallahassee, FL, USA. His current research interests include real-time simulation, renewable energy integration, all-electric-ship power system, and smart grid.

Dr. Faruque is the Chair of the IEEE Power and Energy Society Task Force on Real-Time Simulation of Power and Energy Systems.

ALEXANDRE TENINGE received the Ph.D. degree in electrical engineering from the Grenoble Institute of Technology, Grenoble, France, in 2009.

$\mathrm{He}$ was with the Grenoble Electrical Engineering Laboratory (G2Elab), Grenoble, France, and then with the Laboratory of Electrical Engineering and Power Electronics, Lille, France. He is currently a Research Engineer and the Project Manager with the G2Elab for a smart grid research and development project called SOGRID. His current research interests include fault localization on distribution networks.

ALI HASAN HARIRI (S'14) received the B.Sc. degree in electrical engineering from Lebanese American University, Beirut, Lebanon, in 2013. He is currently pursuing the Ph.D. degree with Florida State University, Tallahassee, FL, USA.

$\mathrm{He}$ is a Research Assistant with the Center for Advanced Power Systems, Florida State University, Tallahassee, FL, USA. His current research interests include the impacts of high penetration PV in distribution networks, integration of DG, power system modeling and simulation, renewable energy, and smart grid research.

LUIGI VANFRETTI (S'03-M'10) received the Electrical Engineering degree from the Universidad de San Carlos de Guatemala, Guatemala City, Guatemala, in 2005, and the M.Sc. and Ph.D. degrees in electric power engineering from the Rensselaer Polytechnic Institute, Troy, NY, USA, in 2007 and 2009, respectively.

He became an Assistant Professor with the KTH Royal Institute of Technology, Stockholm, Sweden, in 2010, where he became a Tenured Associate Professor in 2013. His main research interests include the development of applications of phasor measurement unit data. His current research interests include power system dynamics.

Dr. Vanfretti was conferred the Swedish Title of Docent in 2012.

MARIO PAOLONE (M'07-SM'10) received the Ph.D. degree in electrical engineering with a focus on power systems transients from the University of Bologna, Bologna, Italy, in 2002.

He is currently an Associate Professor with the Swiss Federal Institute of Technology of Lausanne, Lausanne, Switzerland, where he accepted the EOS Holding Chair of the Distributed Electrical Systems Laboratory. His current research interests include real-time monitoring, operation, protections, and transients of power systems.

VENKATA DINAVAHI (S'94-M'00-SM'08) received the Ph.D. degree from the University of Toronto, Toronto, ON, Canada, in 2000.

$\mathrm{He}$ is currently a Professor with the Department of Electrical and Computer Engineering, University of Alberta, Edmonton, AB, Canada. His current research interests include real-time simulation of power systems, large-scale system simulation, and parallel and distributed computing.

PINAKI MITRA (S'07-M'10) received the Ph.D. degree from the Missouri University of Science and Technology, Rolla, MO, USA, in 2010.

$\mathrm{He}$ was an Associate Scientist with the Grid Systems Research and Development Group, ABB, Chennai, India, from 2010 to 2013. Since 2013, he has been with the High Voltage Direct Current (HVdc) Business Unit, ABB Corporate Research, Västerås, Sweden, where he is currently a Senior Research and Development Engineer with the DC Grid Simulation Centre. His current research interests include real-time modeling and control of voltage source converter-based multiterminal HVdc systems and wind integration into the HVdc grid. 
GEORG LAUSS (M'10) received the Degree in mechatronics from Johannes Kepler University Linz, Linz, Austria, in 2006, and the Degrees in scientific thesis from the Eidgenössischen Technischen Hochschule of Zürich, Zürich, Switzerland, and the Université de Paris VI, Paris, France.

$\mathrm{He}$ is currently a Researcher with the AIT Austrian Institute of Technology, Vienna, Austria.

CHRISTIAN DUFOUR (S'88-M'95) received the Ph.D. degree from Laval University, Quebec, QC, Canada, in 2000.

He has been with OPAL-RT Technologies Inc., Montréal, QC, Canada, since 1999, where he is currently a lead Researcher in power systems and motor drive simulation software. He was involved in the development of Hydro-Quebec's HYPERSIM real-time simulator, and MathWorks' SimPowerSystems Blockset.

PAUL FORSYTH (M'95) received the B.Sc. degree in electrical engineering with a focus on electrical power systems from the University of Manitoba, Winnipeg, MB, Canada, in 1988.

He was with $\mathrm{ABB}$ and Haefely-Trench in Europe until he joined RTDS Technologies Inc., Winnipeg, MB, Canada, in 1995.
ANURAG K. SRIVASTAVA (SM'12) received the Ph.D. degree from the Illinois Institute of Technology, Chicago, IL, USA, in 2005.

He joined Washington State University, Pullman, WA, USA, as an Assistant Professor in 2010. His current research interests include power system modeling, operation, and control.

Dr. Srivastava was the Chair of the IEEE Power and Energy Society (PES) Career Promotion Subcommittee and the IEEE PES Student Activities Subcommittee. He is active in several other IEEE PES technical committees, and serves as an Associate Editor of the IEEE Transactions on Smart Grid and the IEEE Distinguished Lecturer.

KAI STRUNZ received the Dr.Ing. (summa cum laude) degree from Saarland University, Saarbrücken, Germany, in 2001.

$\mathrm{He}$ has been a Professor of Sustainable Electric Networks and Sources of Energy with the Technische Universität Berlin, Berlin, Germany, since 2007.

THOMAS STRASSER (M'09-SM'13) received the Ph.D. degree in mechanical engineering with a focus on automation and control theory from the Vienna University of Technology, Vienna, Austria, in 2003.

$\mathrm{He}$ is currently a Senior Scientist with the AIT Austrian Institute of Technology, Vienna, Austria, where he is involved in smart grids with a focus on power utility automation and real-time systems.

ALI DAVOUDI (M'10) received the Ph.D. degree in electrical and computer engineering from the University of Illinois at Urbana-Champaign, Champaign, IL, USA, in 2010.

$\mathrm{He}$ is currently an Assistant Professor with the Department of Electrical Engineering, University of Texas at Arlington, Arlington, TX, USA. 